\title{
Chemical Surface Analysis of Materials: Recent Developments and the Tremendous Growth in Demand for the Technique
}

\author{
Chris Moffitt $^{1}$, Jonathan Counsell ${ }^{2}$, Sarah Coultas $^{2}$ and Nikki Gerrard ${ }^{2}$ \\ ${ }^{1}$ Kratos Analytical, Inc., Corpus Christi, Texas, United States, ${ }^{2}$ Kratos Analytical, Ltd., Manchester, \\ England, United Kingdom
}

The virtual explosion of nano-scale research and functionalization of surfaces for reactions and bonding has greatly increased the need for surface chemical characterization. The surface contribution to the bulk properties dominates when the scale of the particles reaches the point where the near surface is on the order of this same scale. Most bulk characterization techniques have limited sensitivity to surface changes, so a technique that lends itself directly to this scale, such as X-ray Photoelectron Spectroscopy (XPS), has seen demand grow dramatically over the last two decades ${ }^{1}$,

The nature of electron interactions and scattering in the hundreds of electron volt kinetic energy range make the analysis of photo-emitted core level electrons specifically selective to just the outer surface of a material, with the signal decaying exponentially such that $95 \%$ of signal in the mean kinetic energy range will come from less than 10nm from the surface. By changing the collection angle on flat, uniform samples, this surface sensitivity can be increased to the point where the outer $1 \mathrm{~nm}$ dominates the spectral intensity. This sensitivity to the surface of a material, coupled with the ability to remove layers of material, then allows for the collection of composition information with depth.

Until around 10 years ago, material could only be easily removed from inorganic materials, as organic material are not eroded with bombardment with single noble gas ions, but rather turned to mush, destroying chemical and compositional information. The advent of cluster ion technology has now allowed for soft materials to be etched away, revealing pristine chemistry, and now compositional and chemical depth profiling through multiple soft organic layers, to the order of 10um of depth, is possible. Multilayer laminates and adhesives, along with many biological samples, can now be depth profiled, which could not previously be eroded without sustaining dramatic chemical damage.

Developments in electron analyzers and supporting technology have expanded the technique, allowing for, relatively, higher spatial information to be obtained from materials. The inefficiency of the physics process limits the total signal, so that the current scale in photelectron microscopy in the laboratory is restricted to the lateral spatial scale of the order of 1 micron or larger. Mapping and selected area analysis have become routine in the failure analysis regime, but in much of the field spectroscopy still carries the bulk of the information. Hyperspectral imaging has also developed and has shown useful in a number of situations, as subtle chemical changes can be unmasked.

With such a broad range of various materials and physical systems where surface characterization is essential, the technique has proven itself invaluable in multiple current technologies, such a semiconductor, battery technology, solar and fuel cell development, catalysis, medical implants, paper technology, adhesives and coatings, corrosion, and even fabric, hair or contact lens treatment. Examples of the importance of XPS to a wide range of materials systems, and the utility of new developments in cluster ion sources will be discussed.

\section{References}


${ }^{1}$ C. J. Powell, Micros. Today 24, 16 (2016). 